\title{
Automated delineation of thyroid nodules in ultrasound images using spatial neutrosophic clustering and level set
}

\author{
Deepika Koundal*, Savita Gupta, Sukhwinder Singh \\ University Institute of Engineering \& Technology, Panjab University, Chandigarh, India
}

\section{A R T I C L E I N F O}

\section{Article history:}

Received 3 March 2015

Received in revised form 28 October 2015

Accepted 17 November 2015

Available online 28 November 2015

\section{Keywords:}

Thyroid gland

Ultrasound images

Segmentation

Neutrosophic clustering

Computer-aided detection

Level set

\begin{abstract}
A B S T R A C T
An accurate contour estimation plays a significant role in classification and estimation of shape, size, and position of thyroid nodule. This helps to reduce the number of false positives, improves the accurate detection and efficient diagnosis of thyroid nodules. This paper introduces an automated delineation method that integrates spatial information with neutrosophic clustering and level-sets for accurate and effective segmentation of thyroid nodules in ultrasound images. The proposed delineation method named as Spatial Neutrosophic Distance Regularized Level Set (SNDRLS) is based on Neutrosophic L-Means (NLM) clustering which incorporates spatial information for Level Set evolution. The SNDRLS takes rough estimation of region of interest (ROI) as input provided by Spatial NLM (SNLM) clustering for precise delineation of one or more nodules. The performance of the proposed method is compared with level set, NLM clustering, Active Contour Without Edges (ACWE), Fuzzy C-Means (FCM) clustering and Neutrosophic based Watershed segmentation methods using the same image dataset. To validate the SNDRLS method, the manual demarcations from three expert radiologists are employed as ground truth. The SNDRLS yields the closest boundaries to the ground truth compared to other methods as revealed by six assessment measures (true positive rate is $95.45 \pm 3.5 \%$, false positive rate is $7.32 \pm 5.3 \%$ and overlap is $93.15 \pm 5$. $2 \%$, mean absolute distance is $1.8 \pm 1.4$ pixels, Hausdorff distance is $0.7 \pm 0.4$ pixels and Dice metric is $94.25 \pm 4.6 \%$ ). The experimental results show that the SNDRLS is able to delineate multiple nodules in thyroid ultrasound images accurately and effectively. The proposed method achieves the automated nodule boundary even for low-contrast, blurred, and noisy thyroid ultrasound images without any human intervention. Additionally, the SNDRLS has the ability to determine the controlling parameters adaptively from SNLM clustering.
\end{abstract}

(c) 2015 Elsevier B.V. All rights reserved.

\section{Introduction}

The growth of cells or cysts in the thyroid gland leads to nodules which may be solitary, multiple or fused with each other. Generally, the occurrences of malignant cases among all thyroid nodules are $0.1-0.2 \%$ [1]. The frequency of palpable thyroid nodules in adult population is near about $4 \%$ to $8 \%$. It generally increases with the age and affects more than $50 \%$ of the world's population [2]. A thyroid nodule is usually categorized as hypo-echoic, iso-echoic or hyperechoic. Previous studies have shown that hypo-echoic nodules with uneven boundaries are more likely to be evolved into malignant nodules [3]. Though the majority of nodules are benign, few of them may be malignant nodule. Therefore, detection of thyroid nodule

\footnotetext{
* Corresponding author. Tel.: +91 9896809404.

E-mail addresses: koundal@gmail.com (D. Koundal), savitagupta@pu.ac.in (S. Gupta), sukhdalip@pu.ac.in (S. Singh).
}

is aimed to determine a possible malignancy. The malignancy of thyroid nodule's can be assessed by Thyroid Imaging Reporting and Data System (TIRADS) and is categorized as malignant, suspicious for malignancy, borderline, probably benign and benign [2]. Most of the malignant thyroid nodules have distinct histopathological components often merged with surrounding tissues, which make the delineation task difficult [4].

Ultrasound (US) and computerized tomography (CT) are the most commonly used imaging modalities for the detection of thyroid nodules. Primary detection of nodules, such as nodular goiter and thyroid tumors, using US images are well documented in several books and articles [3-5]. Ultrasound image is suitable to detect thyroid nodule due to its vascularity, echogenicity, superficial location, and size. A thyroid ultrasound (TUS) image is blurry and noisy due to artifacts, such as refraction, speckle, acoustic shadowing, and reverberation echo. Segmentation of TUS image in computer-aided detection (CAD) system becomes one of the challenging tasks due to speckle noise and low contrast. One of the major challenges in the 
segmentation of thyroid nodules is to make accurate delineation of nodules within organs due to inherent intensity in-homogeneity of tissue texture and speckle, which appears as bright spots.

In literature, many efforts have been made in nodule delineation using TUS images. Generally, the Active Contour methods using intensity features were used to supervise the contour evolution. One of the models known as Variable Background Active Contour (VBAC) was proposed for nodule detection in TUS images [6-8]. The VBAC model can be employed without pre-processing and is able to detect more than one nodule. It offered more accuracy, topological variations, and edge independency as evaluated to the Active Contour Without Edges (ACWE). A drawback of VBAC is that it is device dependent. In most of the cases, technical skills are required for parameter tuning and time-consuming manual interaction. In spite of this, VBAC is not able to segment isoechoic nodules [8]. ACWE assumed homogeneity for object and background areas. This presumption is violated in TUS images due to the intensity inhomogeneity of the thyroid tissue texture and the presence of calcifications in form of bright spots [9]. Further, Tsantis et al. proposed the hybrid multiscale model (HMM) that integrated the Hough transform and wavelet-based edge detection method for the segmentation of nodules in TUS images [10]. A drawback of the HMM is that it required a priori information about the shape of the nodule boundaries to be detected. Therefore, it was not able to segment benign nodules with elliptical boundaries and malignant nodules which exhibit irregular boundaries. To overcome the drawback of VBAC, Iakovidis et al. integrated the Genetic Algorithm (GA) with VBAC known as GA-VBAC model for nodule segmentation in TUS images with automatic tuning of parameters [11]. Another algorithm named as thyroid boundary detector (TBD) was presented for the detection of thyroid boundaries by providing initial ROI with feature extraction and classification methods in US images [12]. Later, a joint echogenicity-texture (JET) model was presented, which coevaluated the intensity of image and linear binary pattern (LBP) distributions by Mumford-Shah function [15]. The JET model incorporated the advantages of VBAC to delineate hypo-echoic and hyper-echoic nodules as well as iso-echoic nodules. Furthermore, it overwhelmed the drawbacks of HMM through topological adaptability. A limitation of the JET model is that it is not able to distinguish structures such as bigger blood vessels from actual nodules. Later, thyroid nodule detector (TND) system was presented for nodule detection in TUS images and videos with more than $95 \%$ accuracy [16]. One of the thyroid nodule segmentation methods for ultrasound images is given in [17]. The method is consisted of contrast enhancement, smoothing and segmentation based on edge based active contour model. In this method, human intervention is needed for the initialization of active contour model. The performance measures of various thyroid segmentation methods are given in Table 1.

Table 1 reveals the numerous improvements that have been made on CAD of TUS images. The major drawbacks of segmentation methods (such as Support Vector Machine, region growing, etc.) are human interactions such as the pre-labeled ROIs or manually initialized contours. Also, reformulating and training the methods are always time-consuming, especially for complex US images. No work on fully automated thyroid nodule delineation based on unsupervised techniques in ultrasound images is published so far. The echogenicity (such as hypo-, iso- or hyper-echoic), low contrast, non-uniform luminance, speckle noise and other artifacts limit the accuracy of automated delineation of thyroid nodules. Until now the segmentation methods for TUS image are semi-automatic or interactive due to which experts have to initiate, or stopping criteria has to be provided to interrupt the method. To overcome these drawbacks, delineation method has been proposed that performs without any human intervention.
Efforts have been made to automate the segmentation method on ultrasound images using fuzzy clustering to provide rough estimation of contour with the integration of level set method [21-23]. However, the operators are still needed to adjust parameters cautiously for an accurate and effective level set segmentation. The enhanced Fuzzy C-Means (FCM) method with spatial information was given for initialization of level set function [22]. Distance Regularizer Level Set Evolution (DRLSE) method also emerged as a powerful technique in ultrasound segmentation considering its overall performance. But it was not able to track weak edges in US images [24]. DRLSE highly responds on manually initialized contours or pre-labeled ROI, which impedes the automated segmentation of ultrasound image. Hence, an attempt has been made to enhance the DRLSE method in accordance to the application requirements. Neutrosophic based approaches are becoming popular that have been extensively applied to resolve US segmentation problems $[25,26,28-30]$.

As per literature, till date no work is published on level set with neutrosophic clustering for automated nodule delineation in thyroid ultrasound images. In this paper, an integrated scheme named as Spatial Neutrosophic Distance Regularizer Level Set (SNDRLS) is presented for an automatic delineation of nodules in TUS images. Initially, SNLM clustering incorporates spatial information throughout adaptive optimization. Then, parameters of DRLSE are directly obtained from SNLM. Finally, the SNDRLS supervised by SNLM is proposed to delineate the nodules automatically in TUS images. The proposed method is validated on TUS images by comparing its results against delineation from three expert radiologists. Moreover, interpretation of images done by radiologists is subjective. The same dataset of thyroid ultrasound images and comprehensive evaluation metrics are used to compare the performances of different methods.

The rest of the paper is organized as follows. Section 2 presents the materials and methods. Section 3 discusses the experimental results. Discussion and conclusion are given in Sections 4 and 5, respectively.

\section{Material and methods}

\subsection{Image dataset}

As no standard dataset is available on thyroid US images, so previous methods reported in literature were performed by authors on their own dataset acquired from different hospitals for testing and validation. In this research, B-mode thyroid US images of 42 subjects were collected from the Department of Radiology, Post Graduate Institute of Medical Education \& Research (PGIMER), Chandigarh, India for retrospective study. Out of 42 subjects, 16 were males, 26 were females, whose age ranging from 15 to 70 years. The dataset consists of hyper-echoic and hypo-echoic nodules. The images are of size $628 \times 656$ pixels, which were acquired with a 256 gray-level depth using IU22 Philips X Matrix with linear probe at a frequency of $17.5 \mathrm{MHz}$. Each thyroid nodule is outlined by three different experts and the manual delineation is aided as golden standard for comparison. The proposed method was implemented in Matlab 7.9 environment on Toshiba 2.00-GHz Dual-core Laptop.

\subsection{Evaluation metrics}

The metrics used to investigate the performance of the proposed method on TUS images are area based error metrics and boundary based metrics. Area based error metrics are used to evaluate how much nodule pixels are covered by the automated method correctly and how much are covered wrongly. The boundary based 
Table 1

Performance measures of segmentation methods for thyroid ultrasound image.

\begin{tabular}{|c|c|c|c|c|}
\hline Ref. & Year & Method & Measure & Values \\
\hline$[6]$ & 2005 & VBAC & Mean overlap values & $88.8 \%$ \\
\hline [8] & 2007 & VBAC & Overlap value & $94 \pm 0.01 \%$ \\
\hline \multirow[t]{2}{*}{ [10] } & 2006 & HMM & Average accuracy & $91.83 \%$ \\
\hline & & & Mean absolute distance & $90.77 \%$ \\
\hline [11] & 2007 & GA-VBAC & Overlap values & $92.5 \%$ \\
\hline \multirow[t]{4}{*}{ [12] } & 2007 & TBD & Mean accuracy & $93.2 \pm 3.2 \%$ \\
\hline & & LBP & Accuracy & $0.82 \%$ \\
\hline & & $\mathrm{K}-\mathrm{NN}$ & Sensitivity & $0.78 \%$ \\
\hline & & & Specificity & $0.81 \%$ \\
\hline [13] & 2007 & Radon-based & Classification accuracy & $89.4 \%$ \\
\hline \multirow[t]{5}{*}{ [14] } & 2008 & RBF & Accuracy & $96.76 \%$ \\
\hline & & PSO algorithm & Sensitivity & $89.73 \%$ \\
\hline & & & Specificity & $98.59 \%$ \\
\hline & & & PPV & $94.88 \%$ \\
\hline & & & NPV & $97.47 \%$ \\
\hline \multirow[t]{3}{*}[15]{} & 2009 & JET & Overlap & $92.9 \pm 3.9 \%$ \\
\hline & & & Dice & $96.3 \pm 2.1 \%$ \\
\hline & & & Mean absolute distance & $1.2 \pm 0.6$ pixel \\
\hline \multirow[t]{2}{*}{ [16] } & 2010 & TND & Sensitivity & $62 \%$ \\
\hline & & & Specificity & $62 \%$ \\
\hline \multirow[t]{3}{*}{ [17] } & 2010 & SVM & Maximum geometric mean & $90.1 \%$ \\
\hline & & & Sensitivity & $93.8 \%$ \\
\hline & & & Specificity & $86.6 \%$ \\
\hline \multirow[t]{5}{*}{ [19] } & 2009 & Decision tree & Accuracy & $97.5 \%$ \\
\hline & & SVM & Sensitivity & $89.6 \%$ \\
\hline & & & Specificity & $98.5 \%$ \\
\hline & & & NPV & $98.3 \%$ \\
\hline & & & PPV & $89.1 \%$ \\
\hline \multirow[t]{5}{*}{ [20] } & 2010 & Region growing & Accuracy & $96.52 \%$ \\
\hline & & & Sensitivity & $91.58 \%$ \\
\hline & & & Specificity & $97.61 \%$ \\
\hline & & & PPV & $89.14 \%$ \\
\hline & & & NPV & $98.04 \%$ \\
\hline
\end{tabular}

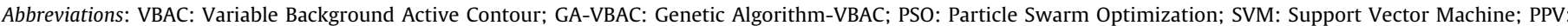
Positive Predictive Value; NPV: Negative Predictive Value; LBP: Linear Binary Pattern.

error metrics are used to measure the possible disagreement over two curves.

a) True positive (TP) is the number of true positive pixels, which are marked as nodule in both automated and manual segmentation. The true positive rate is established by dividing the number of true positives by the total number of nodule pixels in ground truth. The higher percentage value signifies that the segmentation result is closely matched to the expert approximation.

$\mathrm{TP}=\frac{\left|A_{m} \cap A_{a}\right|}{\left|A_{m}\right|} \times 100$

where $A_{a}$ are the pixels automatically generated by the proposed method, and $A_{m}$ are the pixels manually delineated by the expert.

b) False positive (FP) is the number of false positive pixels, which are marked as nodule in automated but not in manual segmentation.

$\mathrm{FP}=\frac{\left|A_{m} \cup A_{a}-A_{m}\right|}{\left|A_{m}\right|} \times 100$

c) Overlap metric $(\mathrm{OM})$ is used for performance evaluation of the proposed method. A higher overlap percentage means an exact match between the two regions.

$\mathrm{OM}=\frac{\left|A_{m} \cap A_{a}\right|}{\left|A_{m} \cup A_{a}\right|} \times 100$

d) Dice coefficient (DC) can be computed as

$\mathrm{DC}\left(A_{a}, A_{m}\right)=\frac{2 \times\left(A_{a} \cap A_{m}\right)}{\left|A_{a}\right|+\left|A_{m}\right|} \times 100$ where $\left|A_{a}\right|$ and $\left|A_{m}\right|$ are the numbers of pixels within the regions $A_{a}$ and $A_{m}$.

e) The Hausdorff distance (HD) is the shortest distance between the segmented and the ground truth boundaries. Let $A_{g}=\left\{a_{g 1}, a_{g 2}\right.$, $\left.\ldots, a_{g m}\right\}$ be the manually annotated ground truth of the nodule contour and $B_{a}=\left\{b_{a 1}, b_{a 2}, \ldots, b_{a n}\right\}$ be the resulting nodule contour from an automatic method. HD is computed as

$\operatorname{HD}\left(A_{g}, B_{a}\right)=\max \left(h\left(A_{g}, B_{a}\right), h\left(B_{a}, A_{g}\right)\right)$,

where $h\left(A_{g}, B_{a}\right)=\max _{a_{g} \in A_{g}} \min _{b_{a} \in B_{a}}\left\|a_{g}-b_{a}\right\|$

where $A_{g}$ and $B_{a}$ are the two curves being compared and $h\left(A_{g}\right.$, $B_{a}$ ) is called Hausdorff distance from $A_{g}$ to $B_{a}$.

f) Mean absolute distance (MAD) is the average distance from a pixel in the segmented boundary to the closest ground truth boundary. MAD is defined as

$\operatorname{MAD}\left(A_{g}, B_{a}\right)=\frac{1}{2}\left(\frac{1}{n} \sum_{i=1}^{n} h\left(A_{g}, B_{a}\right)+\frac{1}{m} \sum_{i=1}^{m} h\left(B_{a}, A_{g}\right)\right)$

\subsection{Methods}

The proposed method is comprised of three steps that are executed automatically: generation of ROI, SNLM clustering and SNDRLS with adapted parameters. Each of these steps acts as an intermediary for the whole method.

\subsubsection{Extraction of ROI}

Several methods have been developed for automatic ROI generation of TUS images $[13,15,20]$. The ROI extraction saves the time from unnecessary processing of irrelevant regions that are not 


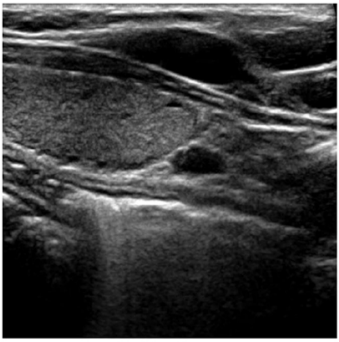

(a)

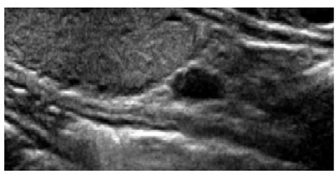

(d)

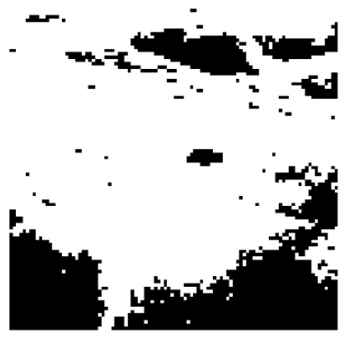

(b)

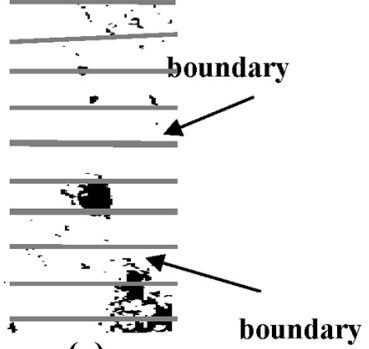

(e)

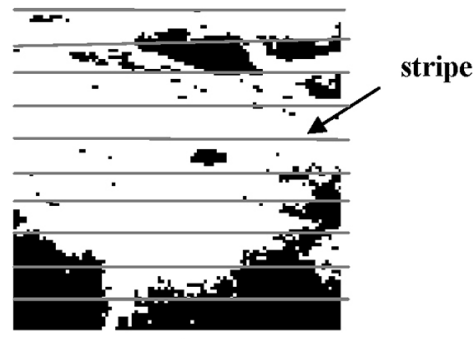

(c)

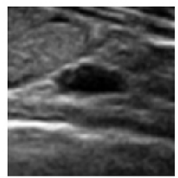

(f)

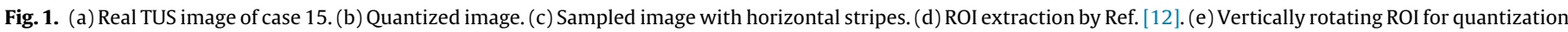
and sampling. (f) Final ROI generated.

required for analysis. The elimination of unimportant regions not only improves the accuracy and execution speed, but also reduces the computational cost. ROI extraction only considers the most likely region for further processing. Here, the ROI extraction is performed by the TBD method mentioned in Ref. [12]. Fig. 1 illustrates the steps of automatic ROI extraction on TUS image for performing further tasks. In the first step, image is normalized and quantized into discrete gray levels (Fig. 1(b)). The quantized image is vertically sampled from top to bottom with non-overlapping and horizontal stripes for rough estimation of hyper-echoic bounds of thyroid gland (Fig. 1(c)). After sampling, the weight and difference of each successive stripe is computed and compared with the threshold value [12].

An index related with the number of pixels of each gray level is calculated for each stripe. The rate of change of each gray level index between two successive stripes is estimated. Finally, the stripes that contain the outer and the inner thyroid boundaries are selected (Fig. 1(d)). Both boundaries should satisfy conditions involving the rates of change of gray level indexes, as well as an additional limitation, which imposes a minimum anteroposterior diameter of the thyroid gland. The details of TBD method for ROI extraction can be found in [12]. The best value of threshold is selected as 0.23 that is searched from a 0.01 step interval [0.1,1] for automatic ROI generation. In second step, transpose the obtained rectangular image and then perform the same operations again, that is, quantization, sampling, and computation of weight of successive stripes (Fig. 1(e)). This will resulted in a final ROI generation (Fig. 1(f)). All further tasks of segmentation are performed on this generated ROI.

\subsubsection{Spatial Neutrosophic L-Means clustering}

Smarandache presented the neutrosophy in 1995 [25]. A pixel in neutrosophic domain $P_{N S}$ is expressed as true component, false component and indeterminacy component. Thus, the degree of true membership ' $T$ ', false membership ' $F$ ' and indeterminacy membership ' $I$ ' is calculated for each pixel in the neutrosophic domain. A nodule can be located in TUS image by considering it as ' $A$ ' background as ' Anti $-A^{\prime}$, and its boundary as ' Neut $-A^{\prime}$. To transform an image into neutrosophic domain, the membership functions $T_{i j}, F_{i j}$ and $I_{i j}$ are defined as

$T_{i j}=1-\hat{g}_{i j}$

$\hat{g}_{i j}=\frac{1}{w_{i} \times w_{i}} \sum_{m=i-\frac{w_{i}}{2}}^{i+\frac{w_{i}}{2}} \sum_{n=j-\frac{w_{i}}{2}}^{j+\frac{w_{i}}{2}} g_{i j}$

$I_{i j}=B_{i j} *\left(1-E_{i j}\right)$

$B_{i j}= \begin{cases}2\left(1-T_{i j}\right) & T_{i j} \geq 0.5 \\ 2 T_{i j} & T_{i j}<0.5\end{cases}$

$F_{i j}=1-T_{i j}$

$E_{i j}= \begin{cases}1 & \text { if } p \text { is on edge } \\ 0 & \text { if } p \text { is not an edge }\end{cases}$

where $T_{i j}, F_{i j}$ and $I_{i j}$ are the True, False and Indeterminate image in neutrosophic domain respectively, $\hat{g}_{i j}$ is the pixel's local mean on a window, $B_{i j}$ is the blur matrix and $E_{i j}$ is the edge matrix [26].

In NLM clustering, membership function and centroid are adaptively approximated to minimize a predefined cost function. The neighboring pixels are highly inter-related to each other due to their identical features, and their probability of belongingness to the same cluster are also high [22]. This spatial association is very significant in NLM. Therefore, an effort has been made to incorporate the spatial relationship into the membership function for accurate image segmentation. The aim of the proposed method is to segment the nodules (foreground) from the background. The proposed SNLM clustering for level set evolution is given in Algorithm 1.

Algorithm 1 (Proposed SNLM clustering method.). Input: Image $f$ Output: Segmented image

Step 1: Initialize $\varepsilon p s=1 e-5$, iteration index $k=0$, membership parameter ' $m$ ', number of clusters ' $L$ ', and membership 
matrix $U^{(k)}=\left[u_{x y}\right]$, where $x$ is the pixel index, $y$ is the cluster index.

Step 2: Transform image into neutrosophic domain by calculating $T^{k}$ and $I^{k}$ for image $f^{k}$ given in Eqs. (7)-(12) and transform $T^{k}$ and $I^{k}$ into vectors VT and VI, respectively, at $k$ th iteration, $k=k+1$.

Step 3: Calculate the center vector $l_{y}$

$l_{y}=\frac{\sum_{x=1}^{N} u_{x y}^{m} \cdot\left(1-V I_{x}\right) \cdot V T_{x}}{\sum_{x=1}^{N} u_{x y}^{m} \cdot\left(1-V I_{x}\right)}$

where $N$ is sum of entire pixels in image, $V T$ and VI are transformed true vector and indeterminate vector, respectively [26]. VI is used to control the contribution of pixels to the cluster centers.

Step 4: (a) Update membership matrix $U^{(k+1)}=\left[u_{x y}\right]$ by

$u_{x y}=\frac{1}{\sum_{d=1}^{L}\left(\frac{V T_{x}-l_{y}}{V T_{x}-l_{d}}\right)^{2 / m-1}}$

(b) Incorporate spatial information using variable $h_{x y}^{q}$

$h_{x y}^{q}=\sum_{k \in N_{n}} u_{y k}$

where $N_{n}$ signifies a window centered around the pixel $n$ in a spatial neutrosophic domain.

(c) Compute spatial membership function $u_{x y}^{\prime}$ by incorporating $h_{x y}^{q}$ directly into the membership function

$u_{x y}^{\prime}=\frac{u_{x y}^{p} h_{x y}^{q}}{\sum_{k=1}^{c} u_{k y}^{p} h_{k y}^{q}}$

where $p$ and $q$ are the two controlling parameters that regulate their respective contribution [21].

Step 5: Update image $f^{(k+1)}$ by

$f^{(k+1)}= \begin{cases}f^{(k)} & \text { if } I^{(k)}<\lambda_{I_{-} T}, \\ \bar{f}^{(k)} & \text { if } I^{(k)} \geq \lambda_{I_{-} T}\end{cases}$

where $\lambda_{I_{-} T}$ is the indeterminacy threshold value.

Step 6: If $\max _{i j}\left\{\left|U_{i j}^{(k+1)}-U_{i j}^{(k)}\right|\right\}<\varepsilon p s$, stop; else go to second step.[18].

The SNLM is a two-pass scheme. Firstly, it determines the distance between cluster centers and pixels to compute the membership function. Then, this membership information is used in spatial domain to compute the spatial function. The spatial function $h_{x y}$ denotes the likelihood of pixel $x_{t h}$ belongs to $y_{\text {th }}$ cluster same as $u_{x y}$ does. The $h_{x y}$ strengthens the membership function and the clustering consequence remains unaffected in homogenous region. The iteration process of SNLM clustering continues with the new membership function incorporating spatial information. The image is updated according to the indeterminacy value, ' $I$ ', which is compared to indeterminacy threshold value, ' $\lambda_{I_{-} T}$ ' as shown in Eq. (17). The description of selecting the best value of $\lambda_{I_{-} T}$ is given in Section 3.4.3. The iterative process is stopped up as the variation between two successive cluster centers is less than $\varepsilon p s$.

\subsubsection{Distance regularized level set evolution (DRLSE)}

The level set method for contour evolution is introduced by Osher and Sethian in 1988 [24]. The concerned contour is embedded as zero level set for level set function (LSF). For accurate computation and stability, LSF is required to be smooth and not too flat or steep during evolution. As a result, re-initialization is needed. Generally in level set, re-initialization is utilized as a numerical solution for evolving sound curve and ensuring consistent results. But the high computational cost is the main problem with reinitialization. DRLSE is used as a generalization of level set evolution to overcome the drawback of re-initialization [24]. This model not only eliminates the re-initialization problem but also reduces its computational complexity.

In DRLSE, a substantial bigger timestep can be employed for providing solution to the evolved partial differential equation, resulting in fast curve evolution. In its formulation, distance regularization and external energy term is utilized that forces the motion of contour toward the desired location accurately. Let $\varphi: \Omega \rightarrow \Re$ be a LSF described on image domain $\Omega$. The gradient flow of energy function $\varepsilon n g(\varphi)$ can be represented as

$\varepsilon n g(\varphi)=\mu \mathscr{R}_{\operatorname{eg}}(\varphi)+\operatorname{En}_{\text {ext }}(g, \varphi)$

where $\mu>0$ is a controlling parameter, $\mathscr{R e g}_{p}(\varphi)$ is the distance regularized term of LSF, $E n_{\text {ext }}$ is the external energy term and $g$ is an edge indicator function [24].

Edge indicator function is used to regularize the force to cease evolution near the best solution which is given as

$g=\frac{1}{1+|\nabla G \sigma * I|^{2}}$

where $G \sigma$ is a Gaussian kernel with a standard deviation $\sigma, I$ be a given $2 \mathrm{D}$ image ${ }^{*}$ is convolution used to smooth the image to reduce the noise, $\nabla$ is the gradient operator. $|\bullet|$ is the modulus of the smoothed image gradients. This function $g$ usually takes smaller values at object boundaries than other locations.

The external energy term can be represented as

$E n_{\text {ext }}(g, \varphi)=\lambda \mathscr{L}_{g}(\varphi)+\alpha \mathcal{A}_{g}(\varphi)$

where $\lambda>0$ and $\alpha \in \Re$ are the coefficients of the energy function $\mathscr{L}_{\mathrm{g}}(\varphi)$ and $\mathcal{A}_{\mathrm{g}}(\varphi)$.

$\mathscr{L}_{\mathrm{g}}(\varphi)=\int \mathrm{g} \delta(\varphi)|\nabla \varphi| d x$

$\mathcal{A}_{g}(\varphi)=\int g H(-\varphi) d x$

where $\delta$ and $H$ are the Dirac delta function and the Heaviside function, respectively.

With Dirac delta function, the energy $\mathscr{L}_{g}(\varphi)$ is used to compute the line integral of the function $g$ and is reduced when zero level contours $\varphi$ is situated at the boundaries of the object. Whereas, $\mathcal{A}_{g}(\varphi)$ is used to accelerate the motion of the zero level contour during evolution, when initial contour is located far-off from the desired boundaries of the object.

DRLSE may be initialized from an arbitrary binary region given as

$\varphi_{0}(x, y)= \begin{cases}-B_{r} & \text { if } \varphi_{0}(x, y) \in \Omega_{0} \\ B_{r} & \text { otherwise }\end{cases}$

where $B_{r}>0$ is a customable constant and $\Omega_{0}$ is a subset in $\Omega$.

The major limitation discovered in the original DRLSE method is the influence of the initial position of curve, which is provided by human intervention.

\subsubsection{Proposed Spatial Neutrosophic Distance Regularized Level Set (SNDRLS)}

Both NLM clustering and DRLSE are computational paradigms that can be employed to solve the image segmentation problems. Image intensity is taken as the supervising trait to enable the delineation of nodules in TUS image. An integrated Spatial Neutrosophic Distance Regularizer Level Set (SNDRLS) method is proposed for automated delineation of nodule in TUS image. The proposed 
method has two phases. Firstly, the SNLM clustering with spatial information is applied to approximate the contours of the nodule region in US image. Subsequently, the results of SNLM are used as input to initialize and regularize the level set by estimating parameters such as $\mu, \tau, \varepsilon, \lambda$, and $\alpha$. In most of the cases, the outcome of segmentation is based on initial position and shape of the initial LSF. If the initial LSF is defined outside of the nodule, then it is likely to be limited at the thyroid's boundary rather than inside of nodule due to weak and strong edges. Thus, appropriate initialization of LSF around the object's boundary is necessary for accurate delineation.

There are various parameters related to DRLSE that are crucial for segmentation of image. Therefore, it is vital to tune them properly. Presently there are few rules for optimum configuration of these parameters. As the larger value of $\sigma$ not only smoothens the image but also some image details are lost. The sign of balloon force $\alpha$ influences the direction of curve evolution. It is essential to select a positive sign of $\alpha$ for shrinkage, if the initial $\varphi$ is outside the object of interest and vice versa. A larger value of time step $\tau$ may lead to boundary leakage while accelerating the DRLSE. It is observed during experiments that larger values of $\lambda$ and $\alpha$ result in smoother contours and accelerate the level set evolution, respectively. The above rules, though effective, are insufficient for optimum parameters configuration of TUS image. Therefore, it is necessary to adapt these controlling parameters by SNLM as given below.

2.3.4.1. Estimation of time step parameter $\tau$. Given the initial level set function $\varphi_{0}^{\text {SNLM }}$ from SNLM clustering, it is convenient to compute time step parameter ' $\tau$ ' as

$\tau=\frac{\alpha}{\ell}$

where $\alpha$ is the weighted area and $\ell$ is the length of initial level set function $\varphi_{0}^{\mathrm{SNLM}}$ obtained from SNLM clustering. Therefore $\tau$ is computed as

$\tau=\frac{\int_{\Omega} g H\left(\varphi_{0}^{S N L M}\right) d x d y}{\int_{\Omega} g \delta\left(\varphi_{0}^{S N L M}\right) d x d y}$

where $H\left(\varphi_{0}^{S N L M}\right)$ is the Heaviside function.

The parameter $\lambda$ controls the topological changes and avoids the boundary leakage problem. To achieve the smooth contour, compute the weighting coefficient of the contour length as

$\lambda=0.1 * \tau$

The product of $\mu^{*} \tau$ should be smaller than $1 / 4$ for numerical stability and accurate evolution [24]. The weighting coefficient of the regularization term is calculated as

$\mu=\frac{0.2}{\tau}$

2.3.4.2. Estimation of parameter $\alpha$. Generally in level set, $\alpha$ is required to be selected as a global constant or controlled for various types of images. A larger $\alpha$ may lead to leakage problem with weak boundaries. In this work, an enhanced balloon force $\alpha$ is introduced to overcome the above drawback. Here, $\alpha$ is the weighted area obtained from initial level set function $\varphi_{0}^{\text {SNLM }}$ that is used to push or pull the dynamic curve adaptively toward the object of interest. The LSF pull toward the object of interest in spite of its preliminary position. It is observed that the initial SNLM can be considered as an index for SNDRLS regularization. The SNDRLS takes the membership degree of each pixel $u_{x y}$ as the distance to the specific object $\mathrm{O}_{k}$.

The proposed method automates the parameter configuration and initialization of level set using SNLM. By incorporating SNLM results directly, the initial curve (LSF) will evolve firmly with its zero level set so that the contour converges to the precise nodule boundary. The object $O_{k}$ obtained by SNLM clustering is closed to the object of interest to be segmented, thus, few iterative steps are required to pull the zero level set from $O_{k}$ to the desired contour. Utilizing the spatial information, a binary image is produced by SNLM clustering that can be represented as

$O_{k}= \begin{cases}1 & u_{x 0}>u_{x 1} \\ 0 & \text { otherwise }\end{cases}$

where $u_{x 0}$ is the foreground membership function and $u_{x 1}$ is the background membership function computed in Eq. (14).

The initial level set function $\varphi_{0}^{\mathrm{SNLM}}$ attained from SNLM is used to estimate the genuine boundaries. Let the object of interest be $O_{k}$ that is used to initiate SNDRLS as

$\varphi_{0}^{\mathrm{SNLM}}=-\varepsilon\left(G\left(O_{k}\right)\right)$

where $\varepsilon$ is the dirac regulator used to smoothen the image and $G\left(O_{k}\right)$ is an improved balloon force to pull or push the curve adaptively toward the $O_{k}$ [21].

$G\left(O_{k}\right)=1-2 O_{k}$

Thus, the external energy equation (Eq. (21)) can be represented as

$E n_{e x t}(g, \varphi)=\lambda \delta_{\varepsilon}(\varphi) \operatorname{div}\left(g \frac{\nabla \varphi}{|\nabla \varphi|}\right)+G\left(O_{k}\right) g \delta_{\varepsilon}(\varphi)$

Finally apply the evolving equation for level set as

$$
\begin{aligned}
\varphi^{k+1}(x, y)= & \varphi^{k}(x, y)+\tau\left[\mu\left[\nabla^{2} \varphi-\operatorname{div}\left(\frac{\nabla \varphi)}{|\nabla \varphi|}\right)\right]\right. \\
& \left.+\lambda \delta_{\varepsilon}(\varphi) \operatorname{div}\left(g \frac{\nabla \varphi}{|\nabla \varphi|}\right)+\left(1-2 O_{k}\right) g \delta_{\varepsilon}(\varphi)\right]
\end{aligned}
$$

With the above procedure, the initialization of SNDRLS by SNLM clustering is completely automatic without any human intervention. This enhancement leads to SNDRLS for nodule segmentation in TUS image with several practical advantages. Here, the SNLM clustering is used to derive the balloon force $\alpha$ directly and the level set evolution is regulated to the distance of the desired object. The evolution will consequentially decelerate as it approaches the object of interest. SNDRLS become stable automatically as conventional $\lambda$ is assumed. With such an enhancement, operator is not required to be careful during evolution, to avoid excessive or inadequate segmentation.

\section{Experimental results and discussion}

\subsection{Performance of SNDRLS}

In this section, the quantitative results of proposed method in terms of six evaluation metrics are illustrated in Figs. 2 and 3 and Table 2. Fig. 2 demonstrates the TP values achieved by the proposed method, as well as the ranges of TP obtained by each individual expert. The average TP value obtained by SNDRLS is $95.45 \pm 3.5 \%$. The minimum value of TP obtained is $89.48 \%$ in case 14 while maximum value of TP is $99.02 \%$ in case 2 .

Fig. 3 demonstrates the FP value achieved by the SNDRLS, as well as the ranges of FP got by experts. The average value of FP obtained is $7.3 \pm 5.3 \%$.

Table 2 presents the values of DC, HD, OM, and MAD achieved by the proposed method. The average value of OM is $93.15 \pm 5.2 \%$ and minimum is $87.9 \%$ in case 33 . Whereas, the average value of DC is $94.25 \pm 4.6 \%$ and minimum is $92.6 \%$ in case 29 . The average value of MAD is $1.8 \pm 1.4$ pixels and maximum value is 3.2 pixels in case 2 . Whereas, the average value of HD obtained is $0.7 \pm 0.4$ 


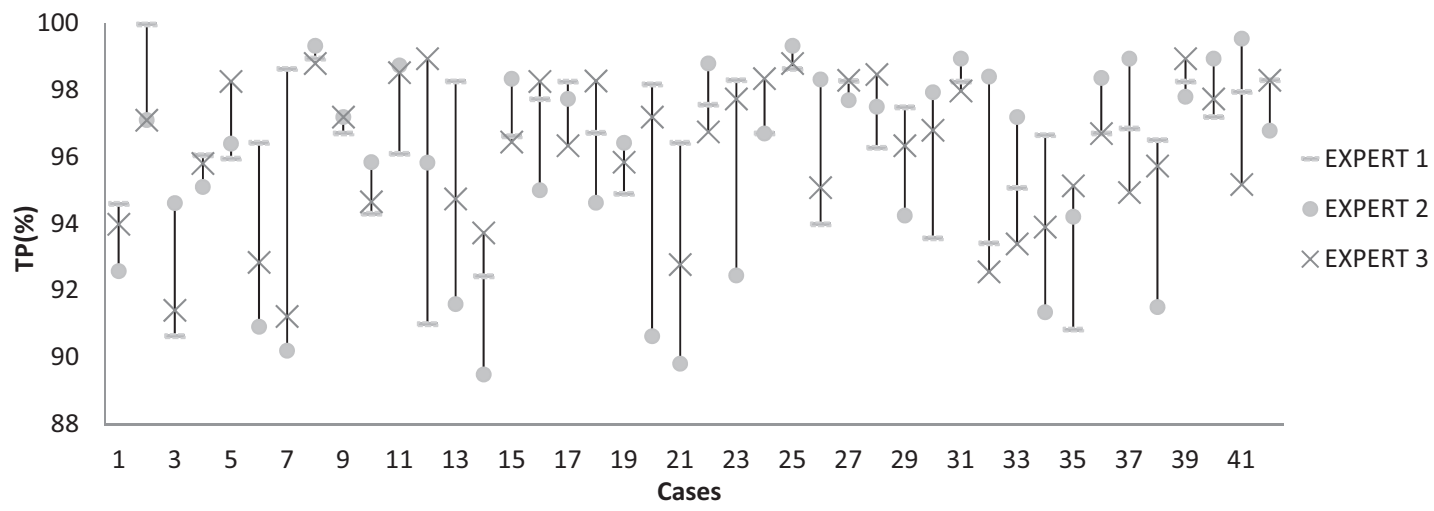

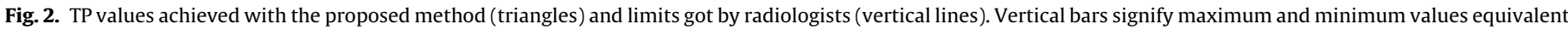
to the regions segmented by the experts.

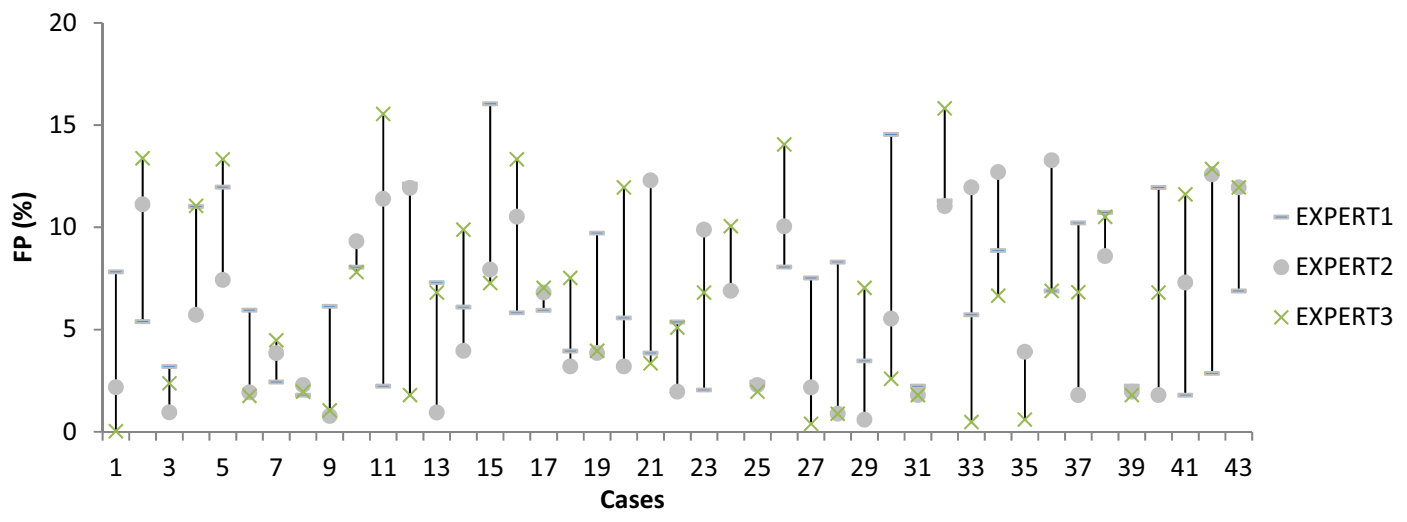

Fig. 3. FP values achieved with the proposed method (triangles) and scales got by experts (vertical lines).

pixels and maximum is 1.1 pixels in case 2 . Moreover, the values of OM exceed $92 \%$ in 37 out of 42 cases and DC exceeds $94 \%$ in 36 out of 42 cases.

The three different component's of neutrosophic image named as true, false and indeterminate are illustrated in Fig. 4(b)-(d), respectively. Whereas, the rough estimation of nodule contour in the form of binary image obtained by SNLM clustering is not connected with any dark region in background illustrated in Fig. 4(e). Fig. 4(f) shows the delineated boundary of nodule in TUS image using the SNDRLS. The contour expands, contracts, splits or merges

Table 2

Proposed Delineation Evaluation Measures.

\begin{tabular}{|c|c|c|c|c|c|c|c|c|c|}
\hline Case & DC (\%) & HD (pixels) & $\mathrm{OM}(\%)$ & MAD (pixels) & Case & $\mathrm{DC}(\%)$ & HD (pixels) & OM (\%) & MAD (pixels) \\
\hline 1 & 98.4 & 0.4 & 97.8 & 0.4 & 22 & 94.3 & 0.4 & 93.8 & 0.8 \\
\hline 2 & 94.7 & 1.1 & 95.1 & 3.2 & 23 & 96.8 & 0.4 & 92.0 & 1.1 \\
\hline 3 & 97.6 & 0.5 & 93.6 & 0.6 & 24 & 95.2 & 0.7 & 93.0 & 2.0 \\
\hline 4 & 92.7 & 0.7 & 96.9 & 1.3 & 25 & 96.4 & 0.6 & 96.7 & 1.1 \\
\hline 5 & 94.9 & 0.8 & 96.0 & 1.1 & 26 & 95.0 & 0.4 & 96.7 & 1.0 \\
\hline 6 & 94.2 & 0.9 & 95.1 & 0.8 & 27 & 97.6 & 0.4 & 95.1 & 1.1 \\
\hline 7 & 97.4 & 0.8 & 97.4 & 1.5 & 28 & 96.2 & 0.4 & 95.5 & 1.4 \\
\hline 8 & 95.6 & 0.5 & 97.0 & 1.1 & 29 & 92.6 & 0.5 & 91.6 & 1.6 \\
\hline 9 & 95.3 & 0.4 & 93.8 & 1.4 & 30 & 96.1 & 0.7 & 95.7 & 0.9 \\
\hline 10 & 93.4 & 0.5 & 97.3 & 0.6 & 31 & 97.9 & 0.6 & 98.4 & 1.1 \\
\hline 11 & 95.8 & 0.3 & 91.5 & 0.5 & 32 & 98.9 & 0.3 & 91.2 & 0.9 \\
\hline 12 & 98.2 & 0.7 & 96.3 & 0.5 & 33 & 93.4 & 0.4 & 87.9 & 0.6 \\
\hline 13 & 95.1 & 0.4 & 93.9 & 0.8 & 34 & 93.3 & 0.4 & 94.3 & 0.5 \\
\hline 14 & 95.2 & 0.4 & 95.7 & 0.4 & 35 & 95.1 & 0.3 & 93.2 & 0.9 \\
\hline 15 & 97.0 & 0.4 & 92.5 & 0.6 & 36 & 94.2 & 0.7 & 94.5 & 2.2 \\
\hline 16 & 95.6 & 0.7 & 94.8 & 1.6 & 37 & 95.9 & 0.5 & 95.3 & 1.1 \\
\hline 17 & 95.5 & 0.4 & 91.4 & 0.9 & 38 & 96.3 & 0.6 & 95.6 & 2.0 \\
\hline 18 & 96.9 & 0.4 & 92.7 & 0.7 & 39 & 94.9 & 0.6 & 96.7 & 1.1 \\
\hline 19 & 93.3 & 0.3 & 93.8 & 0.8 & 40 & 98.5 & 0.4 & 95.1 & 0.7 \\
\hline 20 & 95.0 & 0.4 & 95.8 & 0.7 & 41 & 89.6 & 1.0 & 94.4 & 1.8 \\
\hline 21 & 93.3 & 0.4 & 93.7 & 1.1 & 42 & 95.2 & 0.4 & 94.4 & 1.4 \\
\hline
\end{tabular}

Note: Unit for HD and MD is pixel. 1 pixel is approximately $0.26 \mathrm{~mm}$ for image of DPI = 96 pixels/in. in database. 


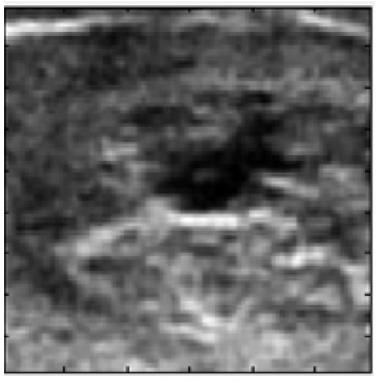

(a)

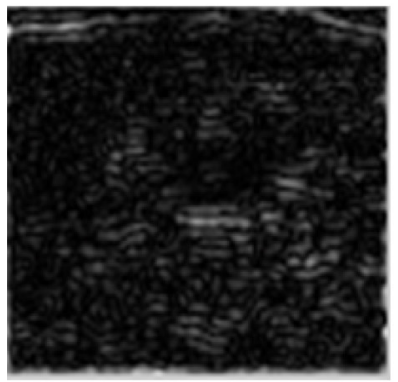

(d)

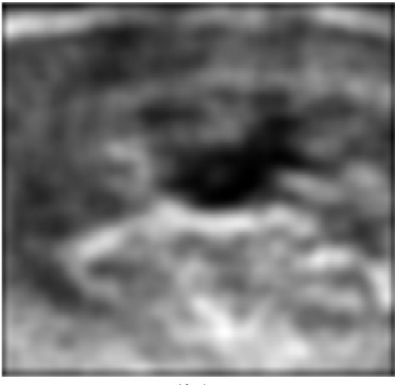

(b)

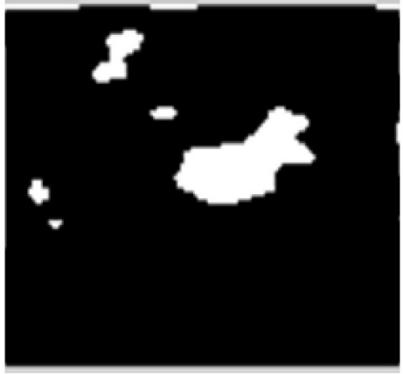

(e)

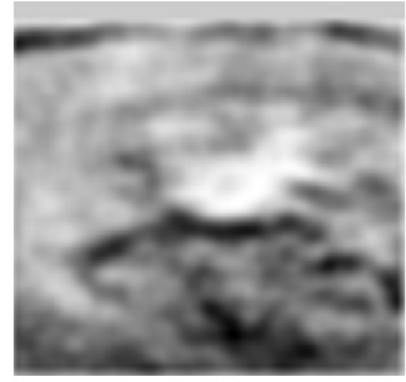

(c)

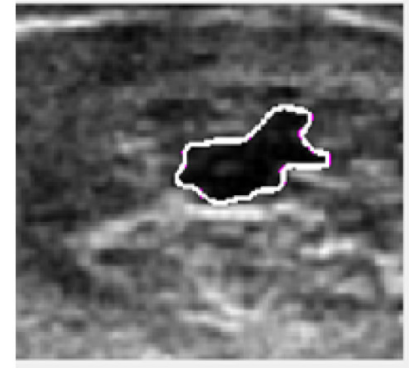

(f)

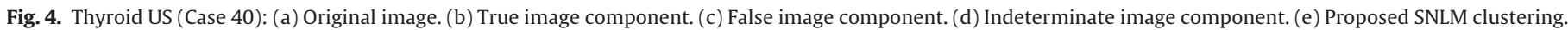
(f) Proposed SNDRLS method.

and stops at the desired location during SNDRLS evolution, resulting in automated and accurate segmentation.

\subsection{Comparison of proposed SNLM method with other segmentation methods}

Six paradigms compared in experiments are illustrated in Fig. 5. Fig. 5(b) shows the outcome of thresholding in which some of the important pixels of the image are cut off. However, it requires cautious selection of optimal threshold value, especially for those edges that are fused to each other. The nodule is not properly segmented out by FCM due to high contrast and weak edges (Fig. 5(c)). The FCM is not so robust due to the presence of artifacts and misclassification of indeterminate pixels into foreground. On the contrary, the output of spatial FCM (Fig. 5(d)) had many false foreground and noisy regions. The spatial FCM cause problems in case of weak and ambiguous boundaries of nodule. The NLM segmented the nodule properly but boundary leakage is there due to weak edges (Fig. 5(e)). The obscure background could be smoothen by NLM but due to lack of spatial information, it is not able to prevent the nodule from joining the false foregrounds. The weak and uneven edges of thyroid nodule often made the segmentation complicated due to intensity inhomogeneity. Fig. 5(f) shows effective and accurate thyroid nodule segmentation in US image. The nodule obtained by proposed SNLM is not connected with any dark region in the background and therefore, nodule boundary is quite clear and accurate. Neutrosophic c-means clustering (NCM) clustering shows an effective segmentation of nodule but has taken considerable time for nodule segmentation (Fig. 5(g)). The quantitative evaluation of proposed SNLM method with Thresholding, FCM [31], spatial FCM [22], NCM [29] and NLM [25] methods in terms of six evaluation metrics and computational time are also given in Table 3.

The delineation accuracies obtained by NLM and SNLM are given in Table 3. The mean values of OM, DC and TP obtained with SNLM are $92.8 \%, 93.8 \%$, and $93.4 \%$, respectively. The mean difference values of OM, DC and TP obtained by the NLM and SNLM methods are $2.7 \pm 0.4,1.4 \pm 1.64$ and $1.6 \pm 3.7$, respectively. In most of the cases, SNLM converge to higher values than NLM and NCM.

\subsection{Comparison of SNDRLS with other methods}

The comparison of proposed SNDRLS with Neutrosophic watershed [27], ACWE [9], Fuzzy Level Set method (FLSM) [21], and DRLSE [24] using six evaluation metrics and execution time on entire dataset is given in Table 4 . The higher TP rate (95.4 $\pm 3.5 \%)$ specifies that the proposed method is able to cover more nodule area than other methods. From Table 4, it is also observed that the proposed method takes less processing time as compared to other methods.

It emphasizes that the average HD (measuring the worst pointdistance between two contours) is obtained as $0.7 \pm 0.4$ pixels and the average mean distance is $1.8 \pm 1.4$ pixels, which means that the contours generated by SNDRLS are much closer to the manual delineations.

The visual results of SNDRLS with other methods are illustrated in Fig. 6. The initial contours are denoted with dashed lines. The parameters used in these methods are well tuned. The ACWE and DRLSE methods got the initial contour of entire thyroid nodule by hand. Manual initialization of DRLSE generated the smoother contour but did not lead to an accurate delineation of nodule due to intensity in-homogeneity and presence of noise (Fig. 6(d)). Further, the ACWE outlined the contour with small noise clusters having large magnitudes of intensity (Fig. 6(f)). Fig. 6(c) and (g) shows that the FCM and neutrosophic watershed method cannot handle the weak boundaries, leading to contour leakage into the surrounding tissues. The FLSM miss-classifies the indeterminate pixels into foreground pixels (Fig. 6(c)). The threshold initialized LSF failed in accurate nodule delineation and had not produced the smooth outline even for the nodule of circular shape (Fig. 6(e)). Fig. 6(h) illustrates that the NLM clustering leaked the weak boundary gradients while the proposed method did not. In contrast, the SNDRLS has the ability to deal with intensity inhomogeneity due to its indeterminacy handling capability. It prevents the boundary leakage into normal tissues due to the use of spatial information (Fig. 6(b)). The results of proposed method demonstrate that the delineation of nodules are better than other methods and are very near to the ground truth. The SNDRLS is beneficial as the implicit boundary becomes stable once it approaches the actual edges. 


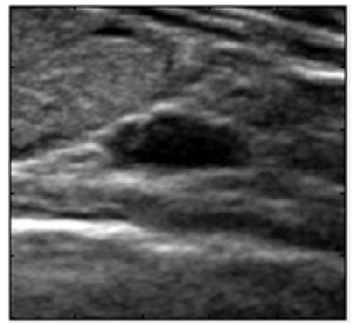

(a)

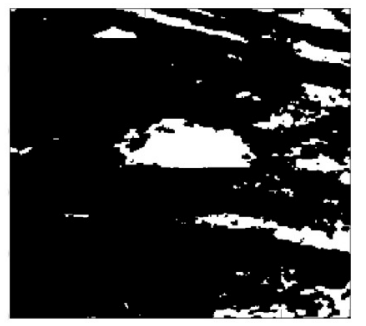

(b)

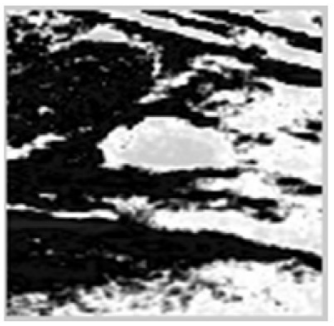

(c)

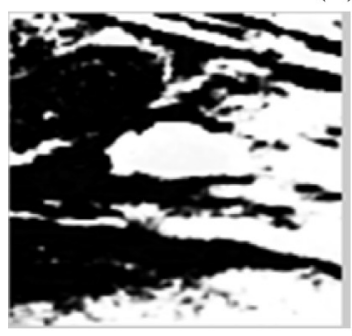

(d)

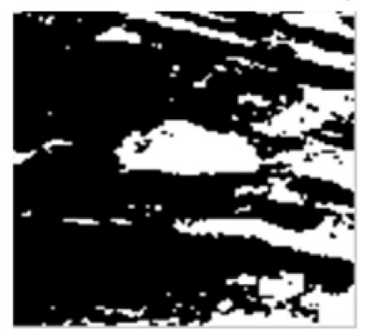

(e)

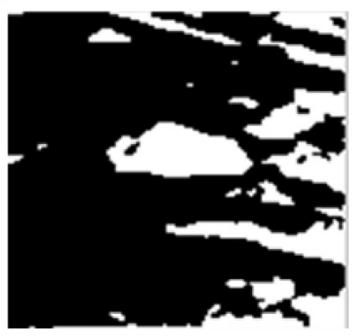

(f)

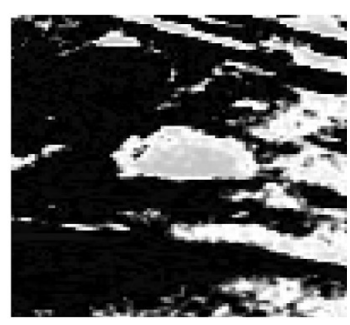

(g)

Fig. 5. (a) Thyroid US image (Case 1): segmentation by (b) thresholding, (c) FCM, (d) spatial FCM, (e) NLM, (f) Proposed SNLM, and (g) NCM.

Table 3

Comparison of proposed SNLM method with other methods.

\begin{tabular}{|c|c|c|c|c|c|c|c|}
\hline Methods & $\mathrm{TP}(\%)$ & $\mathrm{FP}(\%)$ & OM (\%) & DC (\%) & MAD (pixels) & HD (pixels) & Time (s) \\
\hline Thresholding & $71.81 \pm 8.5$ & $5.26 \pm 6.3$ & $70.22 \pm 24.3$ & $81.11 \pm 12.1$ & $0.35 \pm 1.5$ & $0.38 \pm 1.9$ & 0.52 \\
\hline FCM & $89.47 \pm 3.9$ & $37.01 \pm 20.6$ & $68.93 \pm 22.5$ & $71.65 \pm 14.9$ & $1.81 \pm 2.8$ & $0.62 \pm 2.5$ & 5.79 \\
\hline Spatial FCM & $90.15 \pm 4.3$ & $28.13 \pm 15.8$ & $72.64 \pm 18.5$ & $74.03 \pm 16.3$ & $1.91 \pm 1.3$ & $0.64 \pm 2.1$ & 6.10 \\
\hline NCM & $90.07 \pm 7.3$ & $10.44 \pm 5.2$ & $89.14 \pm 10.0$ & $91.55 \pm 8.8$ & $1.40 \pm 0.3$ & $0.66 \pm 0.4$ & 3.10 \\
\hline NLM & $91.80 \pm 6.2$ & $9.90 \pm 8.5$ & $90.1 \pm 7.0$ & $92.4 \pm 4.06$ & $1.90 \pm 1.2$ & $0.90 \pm 0.08$ & 2.10 \\
\hline SNLM & $93.45 \pm 2.5$ & $4.07 \pm 4.8$ & $92.8 \pm 4.6$ & $93.8 \pm 3.7$ & $0.23 \pm 0.9$ & $0.24 \pm 0.8$ & 2.31 \\
\hline
\end{tabular}

Note: Unit for HD and MD is pixel. 1 pixel is approximately $0.26 \mathrm{~mm}$ for image of DPI = 96 pixels/in. in database.

Table 4

Comparison of proposed SNDRLS method with other methods.

\begin{tabular}{|c|c|c|c|c|c|c|c|}
\hline Methods & $\mathrm{TP}(\%)$ & $\mathrm{FP}(\%)$ & $\mathrm{OM}(\%)$ & $\mathrm{DC}(\%)$ & MAD (pixels) & HD (pixels) & Time (s) \\
\hline Neutrosophic watershed & $66.3 \pm 20.1$ & $16.1 \pm 12.5$ & $89.7 \pm 6.6$ & $87.8 \pm 7.2$ & $7.5 \pm 21.3$ & $1.1 \pm 0.7$ & 5.62 \\
\hline ACWE & $67.9 \pm 18.1$ & $6.7 \pm 4.1$ & $75.8 \pm 5.6$ & $76.4 \pm 11.02$ & $1.9 \pm 1.0$ & $0.9 \pm 0.5$ & 6.11 \\
\hline FLSM & $88.5 \pm 5.3$ & $12.07 \pm 11.3$ & $84.7 \pm 7.05$ & $88.7 \pm 7.05$ & $1.68 \pm 0.2$ & $10.28 \pm 0.08$ & 7.72 \\
\hline DRLSE & $89 \pm 3.4$ & $8.7 \pm 2.9$ & $88.3 \pm 5.3$ & $85.6 \pm 5.3$ & $5.47 \pm 3.7$ & $11 \pm 0.06$ & 14.38 \\
\hline SNDRLS & $95.4 \pm 3.5$ & $7.3 \pm 5.3$ & $93.1 \pm 5.2$ & $94.2 \pm 4.6$ & $1.8 \pm 1.4$ & $0.7 \pm 0.4$ & 4.82 \\
\hline
\end{tabular}

Note: Unit for HD and MD is pixel. 1 pixel is approximately $0.26 \mathrm{~mm}$ for image of DPI = 96 pixels/in. in database.

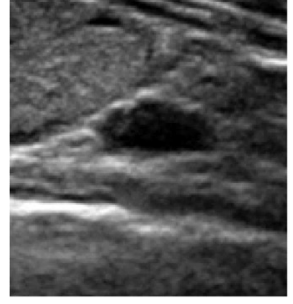

(a)

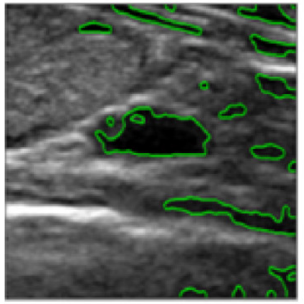

(e)

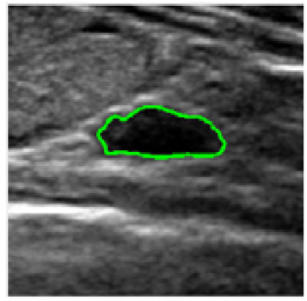

(b)

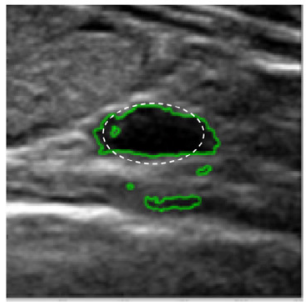

(f)

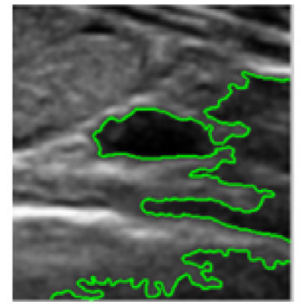

(c)

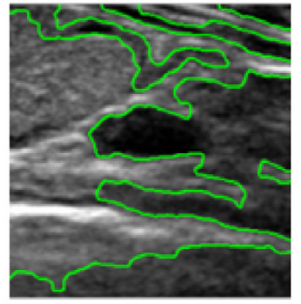

(g)

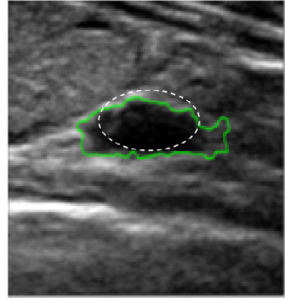

(d)

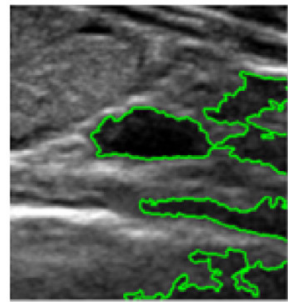

(h)

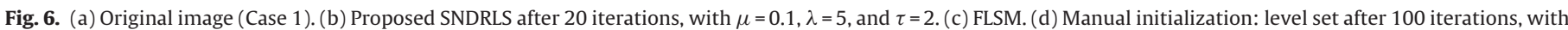

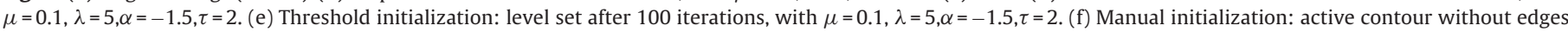
150 iterations, with $\mu=0.1, \lambda=5, \alpha=-1.5, \tau=2$. (g) Neutrosophic watershed. (h) NLM clustering. 
Table 5

Comparison of NDRLS with SNDRLS.

\begin{tabular}{|c|c|c|c|c|c|c|}
\hline Methods & $\mathrm{TP}(\%)$ & $\mathrm{FP}(\%)$ & OM (\%) & $\mathrm{DC}(\%)$ & MAD (pixels) & HD (pixels) \\
\hline NDRLS & $92.3 \pm 4.9$ & $8.4 \pm 4.9$ & $91.9 \pm 5.1$ & $94.5 \pm 2.7$ & $1.9 \pm 1.2$ & $0.9 \pm 0.08$ \\
\hline SNDRLS & $95.4 \pm 3.5$ & $7.3 \pm 5.3$ & $93.1 \pm 5.2$ & $94.2 \pm 4.6$ & $0.2 \pm 0.9$ & $0.2 \pm 0.82$ \\
\hline$p$-value & $<0.005$ & $<0.005$ & $<0.005$ & $<0.005$ & $3.3 \times 10^{-3}$ & $1.9 \times 10^{-9}$ \\
\hline
\end{tabular}

Note: Unit for HD and MD is pixel. 1 pixel is approximately $0.26 \mathrm{~mm}$ for image of DPI $=96$ pixels/in. in database.

The delineation accuracies of Neutrosophic Distance Regularizer Level Set (NDRLS) and SNDRLS are given in Table 5. The mean values of OM, DC and TP obtained with SNDRLS are 93.1\%, 94.2\%, and 95.4\%, respectively. The mean difference values of OM, DC and TP obtained by the NDRLS and SNDRLS methods are $1.2 \pm 0.1,2.97 \pm 2.5$ and $3.1 \pm 1.4$, respectively, in support of SNDRLS. In most of the cases, SNDRLS converge to higher values than NDRLS. The mean difference of the MAD value obtained by the NDRLS and SNDRLS methods are $1.7 \pm 0.3$ pixel, in favor of SNDRLS.

The $p$-value is used to validate the statistical significance of this result. The resulting $p$-value corresponding to each metric is significant in a 0.005 level (Table 5, row 3 ). The irregularity and shape of periphery are the risk factors of thyroid nodule that are considered by an expert for malignancy risks before going to biopsy [8]. Fig. 7 shows the results of proposed method on synthetic image as well as on real thyroid US image with their corresponding mesh, which illustrates that the SNDRLS is able to delineate multiple nodules in US images.

\subsection{Estimation of tunable parameters}

The parameters used in the SNLM are estimated by varying their values in order to obtain optimum value. The tunable parameters in SNLM are $m$ (membership computation), $w$ (window size), and $\lambda_{I_{-} T}$ (threshold of indeterminate value).

\subsubsection{Estimation of window size 'w'}

The controlling parameter $w$ is used as a window size in SNLM method. Different values of window size are chosen to compute different evaluation measures as given in Table 6 . The experiments are performed using window at the size 3,5,7 and 9. By performing different experiments, it is observed that the SNLM is robust as the obtained standard deviation is less than $0.3 \%$ in case of TP, FP, OM, and $\mathrm{DC}$ as well as HD and MAD is less than 0.3 pixels by varying the values of $w$.

\subsubsection{Estimation of membership parameter ' $m$ '}

The parameter $m$ is used to compute membership function. Experiments are performed using different values such as 2, 3, 4 and 5 to compute $m$. From experiments, it is observed that too small standard deviation is achieved by varying the values of $m$ as shown in Table 7. In this work, the value of $m$ is set at 2 to gain optimum results.

\subsubsection{Estimation of indeterminacy threshold parameter ' $\lambda_{\mathrm{I}_{\mathrm{T}}}$ '}

In order to choose an optimum value of indeterminacy threshold, $\lambda_{I_{-} T}$, different values from 0.1 -step interval $[0.1,2.0]$ are taken for different type of images. As shown in Fig. 8, the parameter $\lambda_{I_{-} T}$ is tuned at 1.2 illustrates better results (Fig. 8(b)) as compared to other values (Fig. 8(c-f)). At value 1.2, uncertain and indeterminate pixels are easily distinguishable from background and foreground pixels as noise is reduced iteratively based on indeterminacy threshold.

\section{Discussion}

In this practice, an attempt is made to initialize the LSF automatically with the aid of SNLM clustering. The proposed SNLM incorporates the spatial information with neutrosophic clustering. It is less vulnerable to noise and has the ability to achieve possible nodules adaptively. Formerly, almost all level set methods adopted the manual or threshold techniques for level set initialization. Furthermore, the controller has to be careful to DRLSE in situations of weak or ambiguous boundaries, otherwise excessive or in-sufficient segmentation will happen [24]. In case of DRLSE, $\alpha$ is required to be set according to the image but in SNDRLS, value of $\alpha$ is selected adaptively according to the weighted area

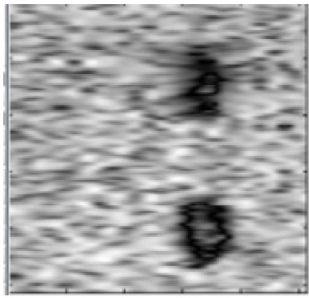

(a)

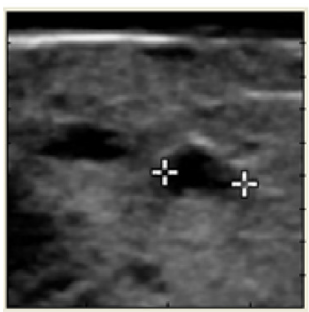

(e)

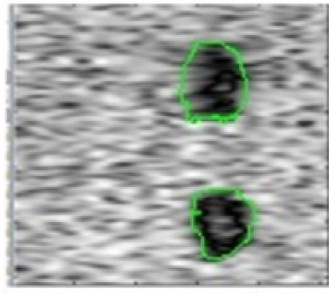

(b)

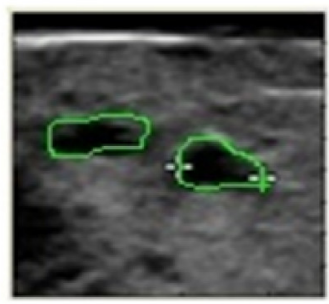

(f)

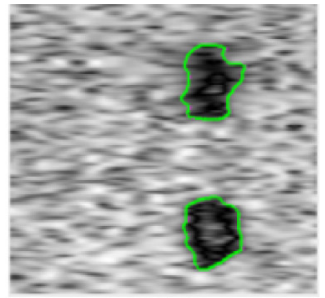

(c)

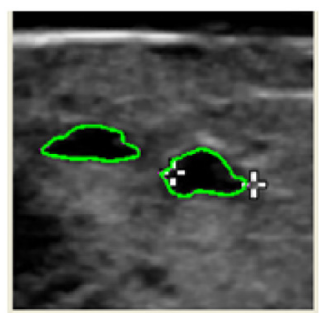

$(\mathrm{g})$

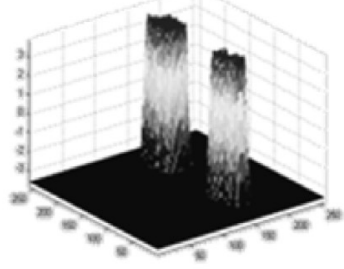

(d)

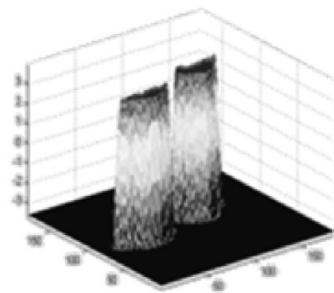

(h)

Fig. 7. (a) original image. (b) Ground truth. (c) SNDRLS. (d) Corresponding 3D level set mesh. 
Table 6

Performance of different values of window size $w$.

\begin{tabular}{|c|c|c|c|c|c|c|}
\hline$w$ & $\mathrm{TP}(\%)$ & $\mathrm{FP}(\%)$ & $\mathrm{OM}(\%)$ & $\mathrm{DC}(\%)$ & Avg. MAD (pixels) & Avg. HD (pixels) \\
\hline 3 & 95.40 & 7.30 & 93.10 & 94.20 & 1.80 & 0.70 \\
\hline 5 & 94.96 & 7.27 & 92.99 & 93.96 & 1.59 & 0.47 \\
\hline 7 & 94.88 & 7.18 & 92.82 & 93.89 & 1.47 & 0.21 \\
\hline 9 & 94.83 & 7.12 & 92.69 & 93.72 & 1.33 & 0.16 \\
\hline Standard deviation & 0.26 & 0.08 & 0.18 & 0.19 & 0.19 & 0.25 \\
\hline
\end{tabular}

Note: Unit for HD and MD is pixel. 1 pixel is approximately $0.26 \mathrm{~mm}$ for image of DPI = 96 pixels/in. in database.

Table 7

Performance of different values of $m$.

\begin{tabular}{|c|c|c|c|c|c|c|}
\hline$m$ & $\mathrm{TP}(\%)$ & $\mathrm{FP}(\%)$ & $\mathrm{OM}(\%)$ & $\mathrm{DC}(\%)$ & Avg. MAD (pixels) & Avg. HD (pixels) \\
\hline 2 & 95.40 & 7.30 & 93.1 & 94.2 & 1.80 & 0.70 \\
\hline 3 & 95.26 & 7.29 & 92.98 & 93.97 & 1.44 & 0.68 \\
\hline 4 & 94.98 & 7.16 & 92.79 & 93.84 & 1.36 & 0.55 \\
\hline 5 & 94.89 & 7.01 & 92.65 & 93.73 & 1.22 & 0.39 \\
\hline Standard deviation & 0.23 & 0.13 & 0.19 & 0.20 & 0.24 & 0.14 \\
\hline
\end{tabular}

Note: Unit for HD and MD is pixel. 1 pixel is approximately $0.26 \mathrm{~mm}$ for image of DPI = 96 pixels/in. in database.

of the object of interest estimated by SNLM. The SNDRLS estimates the optimal parameters automatically from SNLM clustering and becomes stabilized as the implicit interface approaches the particular peripheries. Thus, SNLM gives prior information for SNDRLS evolution.

As shown in Figs. 2 and 3, the average values of TP and FP obtained by SNDRLS are $95.45 \pm 3.5 \%$ and $7.32 \pm 5.3 \%$, respectively, with respect to three experts. The proposed method has achieved very low FP values showing its low ratio of false segmentation. The average values of OM, MAD, HD, and DC are $93.15 \pm 5.2 \%, 1.8 \pm 1.4$ pixels, $0.7 \pm 0.4$ pixels, and $94.25 \pm 4.6 \%$, respectively, given in Table 2. Moreover, SNDRLS has the highest DC and OM which indicates that the contour delineated by the proposed method fits to the nodule boundaries accurately among other methods. The highest similarity is further verified by both the MAD and HD measures. The percentage agreements between the automatic and the manual delineated boundaries are within the inter-observer range. Hence, the proposed SNDRLS method achieves the best overall performance.
From Fig. 5(a), it is clear that the image is of poor contrast and has considerable noise and weak edges. The FCM is also unable to delineate nodules accurately, the contour leaks out through weak edges as shown in Fig. 5(c). Fig. 5(e) illustrates that NLM is not able to guess the nodule boundary in case of diffused edges between nodules and surrounding tissue, thus leaves this important clinical information for the physician. Whereas, SNLM is able to segment the nodule properly due to its indeterminacy handling capability (Fig. 5(f)). The application of the SNLM is satisfactory, allowing the correct distinction of the nodule from other near edges and small features, which greatly facilitates the detection of nodule boundaries for the estimation of initial contour. It is worth noting that threshold initialized LSF for the image shown in Fig. 6(e) is highly irregular, and so are the zero level contours. Furthermore, the methods shown in Fig. 6(e) and (g) are sensitive to speckle noise, and the contour detected by these methods are quite irregular except SNDRLS. From Fig. 6(b), it is observed that the proposed method accurately captures the nodule contour despite of different contrast levels and speckle artifacts.

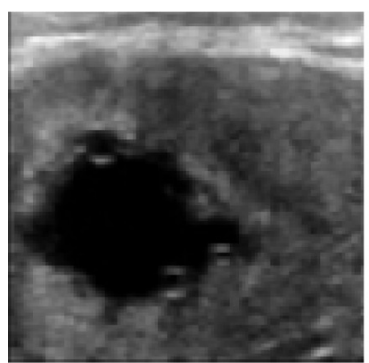

(a)

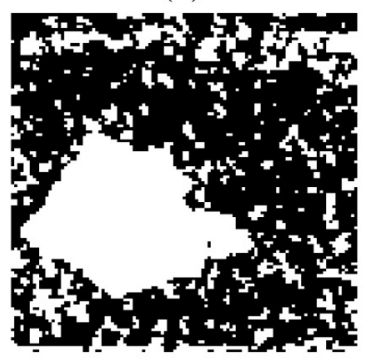

(d)

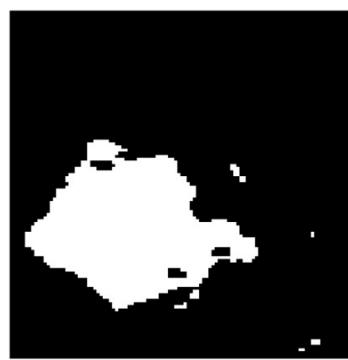

(b)

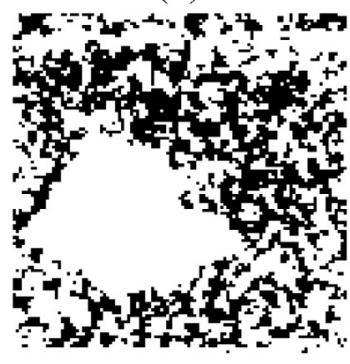

(e)

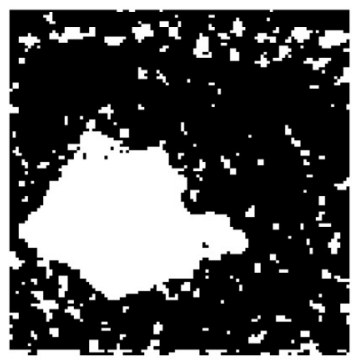

(c)

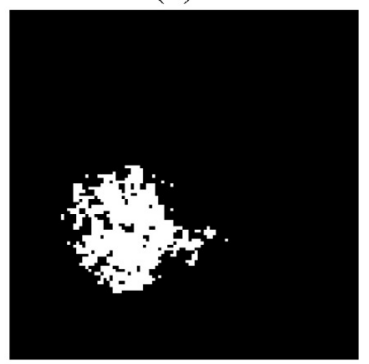

(f)

Fig. 8. (a) Original ultrasound. Proposed SNLM clustering at indeterminacy threshold. (b) $\lambda_{I_{-} T}=1.2$. (c) $\lambda_{I_{-} T}=1.1$. (d) $\lambda_{I_{-} T}=1$, (e) $\lambda_{I_{-} T}=0.9$. (f) $\lambda_{I_{-} T}=0.8$. 
In all experiments, initial contours are successfully provided by SNLM for hyper-echoic and hypo-echoic nodules, i.e., the contours are always very close to the true nodule boundaries of the thyroid. The major advantages of proposed method is that it can cope with indeterminate intensity pixels effectively and accurately. It can smooth the vague background; therefore, it can prevent the boundary of nodule from connecting false foregrounds. Besides the above major advantages, SNDRLS is also able to find nodules even in noisy and complicated cases as well as capable of segmenting more than one nodule (Fig. 7). Also, Table 5 demonstrates the statistically significant difference between two methods to show that their application is feasible in clinical practice otherwise clinical decisions depend on patient and nature of decisions being made. Tables 6 and 7 present the SNDRLS robustness for the processing of ultrasound images. Fig. 8 illustrates that SNLM is able to remove indeterminacy, uncertainty and fuzziness of pixels. From experimental results, it is observed that the proposed method is effective and accurate.

The experiments conducted on the TUS images demonstrate that the SNDRLS outperforms the other methods in in-homogeneous regions. The proposed method is unaffected by speckle noise and able to isolate all nodules even with unclear and blurry edges. It may act as a second opinion tool for automated and accurate delineation of nodules in thyroid ultrasound images. The performance of SNDRLS on new dataset is unknown and may vary substantially from the results reported in this work. Therefore, in future, the SNDRLS will be applied on different image datasets acquired by some other machines.

\section{Conclusion}

In this paper, an integrated method named as Spatial Neutrosophic Distance Regularizer Level Set is proposed for automated delineation of nodules in TUS images. The method integrates the benefits of SNLM clustering and level set, involving multiple nodules delineation and noise robustness capabilities. Additionally, it handles the intensity in-homogeneity taken into account for background information that changes during iterations. Thus, it is proficient in revealing thyroid nodule regardless of intensity variations. The method can be applied without preprocessing due to its indeterminacy handling capability. The improved values of TP, DC, $\mathrm{HD}, \mathrm{OM}$, and MAD are achieved by the proposed method with that of experts and other methods which recommend that its application in medical practice is viable. Additionally, the proposed SNDRLS does not require any training by the user. The only input required is a rough region of interest provided by SNLM within the thyroid gland.

In future, the work can be extended by including texture features to delineate iso-echoic thyroid nodules as well as developing a classifier that can distinguish benign and malignant nodules based on the features extracted from the segmentation result. This method has focused on the segmentation of nodule from thyroid US images. Other modalities such as CT and MRI are still need to be explored with this proposed method.

\section{Acknowledgement}

The author would like to thank the anonymous reviewers for their valuable comments in improving the quality of this manuscript. We are also thankful to the PGIMER, Chandigarh, India for providing image dataset and radiologists for marking the nodules in thyroid US images. The authors would like to thank Y. Guo for providing code of NCM [29].

\section{References}

[1] A.G. Unnikrishnan, U.V. Menon, Thyroid disorders in India: an epidemiological perspective, Indian J. Endocrinol. Metab. 15 (Supplement 2) (2011) 78-81.

[2] E. Horvath, S. Majlis, R. Rossi, C. Franco, J.P. Niedmann, A. Castro, An ultrasonogram reporting system for thyroid nodules stratifying cancer risk for clinical management, J. Clin. Endocrinol. Metab. (2009) 748-751.

[3] D. Koundal, S. Gupta, S. Singh, Survey of computer-aided diagnosis of thyroid nodules in medical ultrasound images, in: Proceedings of the Second International Conference on advances in Computing and information Technology (ACITY) AISC 177, vol. 2, Springer-Verlag, Berlin/Heidelberg, 2012, pp. 459-467.

[4] K. Ain, M.S. Rosenthal, The Complete Thyroid Book, Second edition, McGraw Hill, 2011.

[5] Deepika Koundal, Savita Gupta, Sukhwinder Singh, Computer-aided diagnosis of thyroid nodule: a review, Int. J. Comput. Sci. Eng. Surv. 3 (4) (2012) 67-83.

[6] M. Savelonas, D. Maroulis, D. Iakovidis, S. Karkanis, A Variable Background Active Contour Model for Automatic Detection of Thyroid Nodules in Ultrasound Images, IEEE, 2005, pp. 1-4.

[7] D.E. Maroulis, M.A. Savelonas, S.A. Karkanis, D.K. Iakovidis, N. Dimitropoulos, Computer-aided thyroid nodule detection in ultrasound images, in: Proceedings of the 18th IEEE Symp. on Computer-Based Medical Systems, 2005, pp. 1-6.

[8] D.E. Maroulis, M.A. Savelonas, D.K. Iakovidis, S.A. Karkanis, N. Dimitropoulos, Variable background active contour model for computer-aided delineation of nodules in thyroid ultrasound images, IEEE Trans. Inf. Technol. Biomed. 11 (2007) 537-543.

[9] T.F. Chan, L.A. Vesse, Active contours without edges, IEEE Trans. Image Process. (2001) 266-277.

[10] S. Tsantis, N. Dimitropoulos, D. Cavouras, G. Nikiforidis, A hybrid multi-scale model for thyroid nodule boundary detection on ultrasound images, Comput. Methods Prog. Biomed. (2006) 86-98.

[11] D.K. Iakovidis, M. Savelona, S.A. Karkanis, D. Maroulis, A Genetically Optimized Level Set Approach to Segmentation of Thyroid Ultrasound Images, Springer, 2007, pp. 193-203.

[12] E.G. Keramidas, D.K. Iakovidis, D. Maroulis, S. Karkanis, Efficient and effective ultrasound image analysis scheme for thyroid nodule detection, ICIAR, LNCS 4633 (2007) 1052-1060.

[13] M.A. Savelonas, D.K. Iakovidis, N. Dimitropoulos, D. Maroulis, Computational characterization of thyroid tissue in the radon domain, in: IEEE International Symposium on Computer-Based Medical Systems, 2007, pp. 1-4.

[14] C. Chang, Y. Lei, C. Tseng, S. Shih, Thyroid segmentation and volume estimation in ultrasound images, in: IEEE Int. Conf. on Systems, Man and Cybernetics, 2008, pp. 3442-3447.

[15] M.A. Savelonas, D.K. Iakovidis, I. Legakis, D. Maroulis, Active contours guided by echogenicity and texture for delineation of thyroid nodules in ultrasound images, IEEE Trans. Inf. Technol. Biomed. 13 (2009) 519-527.

[16] E.G. Keramidas, D. Maroulis, D.K. Iakovidis, TND: a thyroid nodule detection system for analysis of ultrasound images and videos, J. Med. Syst. (2010) 1-11.

[17] J. Ma, S. Luo, M. Dighe, D. Lim, Y. Kim, Differential diagnosis of thyroid nodules with ultrasound elastography based on support vector machines, in: IEEE Int. Ultrasonics Symp. Proceedings, 2010, pp. 1372-1375.

[18] P. Ganesh, J. Jai Jaganath Babu, Suganth S. Kannan, Automated thyroid nodule segmentation algorithm for ultrasound images, Int. J. Adv. Res. Electr. Electron. Instrum. Eng. 3 (Special Issue 3) (2014).

[19] C.Y. Chang, H. Huang, S. Chen, Thyroid nodule segmentation and component analysis in ultrasound images, in: Proceedings of APSIPA Annual Summit and Conf., Sapporo, Japan, 2009, pp. 910-917.

[20] C.Y. Chang, Y. Lei, C. Tseng, S. Shih, Thyroid segmentation and volume estimation in ultrasound images, IEEE Trans. Biomed. Eng. 57 (2010) 1348-1357.

[21] B.N. Li, C.K. Chui, S. Chang, S.H. Ong, Integrating spatial fuzzy clustering with level set methods for automated medical image segmentation, Comput. Biol. Med. 41 (2011) 1-10.

[22] K.S. Chuang, H.L. Hzeng, S. Chen, J. Wu, T.J. Chen, Fuzzy c-means clustering with spatial information for image segmentation, Comput. Med. Imaging Graph. 30 (2006) 9-15.

[23] S. Ho, E. Bullitt, G. Gerig, Level set evolution with region competition: automatic 3-D segmentation of brain tumors, in: Proceedings of the International Conference on Pattern Recognition (ICPR'02), 2002, pp. 532-535.

[24] C. Li, C. Xu, C. Gui, M.D. Fox, Distance regularized level set evolution and its application to image segmentation, IEEE Trans. Image Process. 19 (12) (2010).

[25] F. Samarandache, A unifying field in logics Neutrosophic logic, in: Neutrosophy. Neutrosophic Set, Neutrosophic Probability, 3rd edition, American Research Press, 2003.

[26] J. Shan, H.D. Cheng, Y. Wang, A novel segmentation method for breast ultrasound images based on neutrosophic l-means clustering, Med. Phys. 39 (9) (2012) 5669-5682.

[27] M. Zhang, L. Zhang, H.D. Cheng, A neutrosophic approach to image segmentation based on watershed method, Signal Process. 90 (2010) 1510-1517.

[28] Y. Guo, A. Sengur, A novel image segmentation algorithm based on neutrosophic filtering and level set, Neutrosophic Sets Syst. 1 (2013) 46-49.

[29] Y. Guo, A. Sengur, NCM: neutrosophic c-means clustering algorithm, Pattern Recognit. 48 (8) (2015) 2710-2724.

[30] Y. Guo, A. Sengur, NECM: neutrosophic evidential c-means clustering algorithm, Neural Comput. Appl. 26 (3) (2015) 561-571.

[31] S. Kirindis, V. Chatzis, A robust fuzzy local information C means clustering algorithm, IEEE Trans. Image Process. 19 (5) (2010) 1328-1337. 